\title{
ESTIMATES FOR OPERATOR NORMS ON WEIGHTED SPACES AND REVERSE JENSEN INEQUALITIES
}

\author{
STEPHEN M. BUCKLEY
}

\begin{abstract}
We examine the dependence on the $A_{p}$ norm of $w$ of the operator norms of singular integrals, maximal functions, and other operators in $L^{p}(w)$. We also examine connections between some fairly general reverse Jensen inequalities and the $A_{p}$ and $R H_{p}$ weight conditions.
\end{abstract}

\section{INTRODUCTION}

A question of considerable interest in harmonic analysis is, "What types of weights $w$ have the property that $T$ is bounded on $L^{p}(w)$ ?" where $1<p<\infty$, and $T$ is an operator which is bounded on the (unweighted) space $L^{p}$ (typically $T$ is the Hardy-Littlewood maximal operator, singular integral operators, or various related operators of interest in harmonic analysis). This type of question has been answered to a large extent by the work of Muckenhoupt, Hunt, Wheeden, Coifman, C. Fefferman, and others. In particular, it is known that Muckenhoupt's $A_{p}$ condition is a necessary and sufficient condition for boundedness in the case of the Hardy-Littlewood maximal operator or singular integral operators (see $[15,13$, and 4]). However, the dependence of the resulting operator norms on the "badness" of the $A_{p}$ weight has never been adequately examined. We carry out this investigation in $\S 2$, where we also give a new proof of the boundedness of the Hardy-Littlewood maximal operator on $L^{p}(w)$, for $w \in A_{p}$.

$A_{p}$ and $R H_{p}$ conditions are particular types of "reverse Jensen" inequalities which hold uniformly for all cubes. In $\S 3$, we examine more general reverse Jensen inequalities (which hold uniformly for all cubes) with respect to some doubling measure $\mu$ on $\mathbf{R}^{n}$, and show how they are related to the usual $A_{p}(d \mu)$ and $R H_{p}(d \mu)$ conditions. Let us now introduce some notation and give some basic definitions.

For any set $S \subset \mathbf{R}^{n},|S|$ is the Lebesgue measure of $S$. We will use the term "weight" to refer to any nonnegative locally integrable function which is

Received by the editors December 18, 1990 and, in revised form, May 10, 1991 and September 3, 1991.

1980 Mathematics Subject Classification (1985 Revision). Primary 42B20, 42B25.

Key words and phrases. Singular integral, maximal function, weights.

The material in this work was drawn mainly from the author's Ph.D. thesis which was done under the supervision of Robert Fefferman at the University of Chicago.

(C) 1993 American Mathematical Society $0002-9947 / 93 \$ 1.00+\$ .25$ per page 
not everywhere zero. For any measure $\mu$, we write

$$
f_{S} g d \mu \equiv \frac{1}{\mu(S)} \int_{S} g d \mu
$$

(if $\mu$ is Lebesgue measure, we write $g_{S} \equiv f_{S} g$ ). If $w$ is a weight, we will write $w(S)=\int_{S} w$. By a "cube" in $\mathbf{R}^{n}$, we will mean an $n$-fold product of intervals of equal length (i.e. every face of the cube is perpendicular to a coordinate axis). If $Q$ is a cube, $r Q$ will denote the cube concentric with $Q$ whose sidelength is $r$ times that of $Q$ (the " $r$-fold dilate" of $Q$ ). $w$ will always denote a weight on $\mathbf{R}^{n}$ and $p$ is a real number in the range $(1, \infty)$, unless otherwise stated. For any positive quantities $X, Y$, " $X \sim Y$ " will mean " $1 / C \leq X / Y \leq C$ ", where $C$ is independent of the weight $w$ (but may depend on $n, p$, and the operator $T)$. For any exponent $p, p^{\prime}$ denotes the dual exponent $p /(p-1)$.

Definition. A singular integral operator is a principal value convolution operator $T: f \rightarrow K * f$ in $\mathbf{R}^{n}$, where the real-valued kernel $K$ satisfies the following size and cancellation conditions:

$$
\begin{gathered}
\|\widehat{K}\|_{\infty} \leq C, \quad|K(x)| \leq C /|x|^{n}, \\
|K(x)-K(x-y)| \leq C|y| /|x|^{n+1} \quad \text { for }|y|<|x| / 2 .
\end{gathered}
$$

$T^{*}$ denotes the associated maximal singular integral operator which is defined by

$$
T^{*} f(x)=\sup _{\varepsilon>0}\left|\left(K \cdot \chi_{\mathbf{R}^{n} \backslash B(0, \varepsilon)}\right) * f(x)\right|
$$

Definition. If $\mu$ is a positive measure on $\mathbf{R}^{n}$, we say $w$ is an $A_{p}(d \mu)$ weight (we write $w \in A_{p}(d \mu)$ ) if there is some $K>0$ such that for all cubes $Q \in \mathbf{R}^{n}$,

$$
\left(f_{Q} w d \mu\right)\left(f_{Q} w^{-1 /(p-1)} d \mu\right)^{p-1} \leq K
$$

We say $w$ is an $A_{1}(d \mu)$ weight if, for all cubes $Q \in \mathbf{R}^{n}$,

$$
f_{Q} w d \mu \leq K \underset{x \in Q}{\operatorname{essinf}} w(x) \text {. }
$$

The smallest $K$ for which (1.1) (or (1.2)) is true is referred to as the $A_{p}(d \mu)$ norm of $w$ and will be denoted $K_{w, p ; \mu}$ (resp. $K_{w, 1 ; \mu}$ ) or simply $K_{w, \mu}$. We also write $\sigma$ in place of $w^{-1 /(p-1)}$ and refer to $\sigma$ as the dual weight of $w$. It is easy to see that $w \in A_{p}(d \mu)$ if and only if $\sigma \in A_{p^{\prime}}(d \mu)$, and that $K_{\sigma, p^{\prime} ; \mu}=$ $K_{w, p ; \mu}^{p^{\prime}-1}$. It is also clear that $w \in A_{1}(d \mu)$ if and only if $M_{\mu} w \leq K w \quad\left(M_{\mu}\right.$ indicates the Hardy-Littlewood maximal operator with respect to the measure $\mu)$.

Definition. We say $w$ is an $A_{\infty}(d \mu)$ weight if, for all cubes $Q$, and all $E \subset Q$, we have

$$
\frac{\nu(E)}{\nu(Q)} \leq C\left(\frac{\mu(E)}{\mu(Q)}\right)^{\varepsilon}
$$

for some $C, \varepsilon>0$, where $d \nu=w d \mu$. 
Until $\S 3$, we are interested only in $\mu=$ Lebesgue measure, and so we suppress references to $\mu$ (i.e. we write $A_{p}, K_{w}$, etc.). Weights of the form $w_{r}(x)=|x|^{r}$, the so-called power weights, provide the most basic examples of $A_{p}$ weights; in fact $w_{r} \in A_{p}\left(\mathbf{R}^{n}\right)$ if and only if $-n<r<n(p-1)$. We have the following more precise estimates (the proof is straightforward and so we omit it).

Lemma 1.4. If $0<\delta<1$, then $u(x) \equiv|x|^{-n(1-\delta)} \in A_{1}$ and $K_{u, p} \sim 1 / \delta$, for any $p \geq 1$; also, $v(x) \equiv|x|^{n(p-1)(1-\delta)} \in A_{p}$ and $K_{v, p} \sim 1 / \delta^{p-1}$.

It is easy to prove that the dual space of $L^{p}(w)$ is $L^{p^{\prime}}(\sigma)$. In addition, we have the following useful lemma, whose easy proof we also omit.

Lemma 1.5. If a singular integral operator $T$ is bounded on $L^{p}(w)$ and on $L^{p^{\prime}}(\sigma)$ for some $1<p<\infty$, then the two associated operator norms of $T$ are equal.

\section{BOUNDS FOR OPERATOR NORMS}

In this section, $C$ will denote a generic positive constant independent of everything, except possibly the dimension $n$, exponent $p$, and operator $T$. Also, for any weights given as examples, $\delta$ will denote a positive quantity which tends to 0 .

We now look at several important operators which are bounded on $L^{p}(w)$ spaces iff $w \in A_{p}$, and examine how the resulting operator norms depend on $K_{w}$, the $A_{p}$-norm of $w$. Our first main goal will be to do this for the HardyLittlewood maximal operator. We give a new proof of boundedness which gives a best possible dependence estimate. First of all, we need a few preparatory lemmas.

Lemma 2.1 [4]. If $w \in A_{p}$, then $w \in A_{p-\varepsilon}$, where $\varepsilon \sim K_{w, p}^{1-p^{\prime}}$, and $K_{w, p-\varepsilon} \leq$ $C K_{w, p}$.

The next lemma, due to Besicovitch [1], is commonly referred to as the Besicovitch covering lemma. A proof of it can be found in [11, pp. 2-5]. Note that (ii) and (iii) just say that the sequence of cubes can be distributed into a bounded number of disjoint families.

Lemma 2.2. Suppose that $A \subset \mathbf{R}^{n}$ is bounded and that for each $x \in A, Q_{x}$ is a cube centered at $x$. Then we can choose, from among $\left\{Q_{x}: x \in A\right\}$, a (possibly finite) sequence $\left\{Q_{i}\right\}$ and an associated sequence of integers $\left\{m_{i}\right\}$ such that

(i) $A \subset \bigcup_{i} Q_{i}$.

(ii) $1 \leq m_{i} \leq N_{n}$, where $N_{n}$ depends only on $n$.

(iii) $Q_{i}$ and $Q_{j}$ are disjoint if $m_{i}=m_{j}$.

We say an operator is of weak-type $p$, with respect to the measure $\mu$, if

$$
\mu(\{T f>\alpha\}) \leq\left(\frac{C\|f\|_{L^{p}(d \mu)}}{\alpha}\right)^{p} .
$$

The smallest such $C$ is referred to as weak-type $L^{p}(d \mu)$-norm of $T$. We can now state a precise version of the Marcinkiewicz interpolation theorem with respect to a positive measure $\mu$ (the statement of this result given here, for $\mu$ being Lebesgue measure, is a special case of the result as proved by Zygmund [18]). 
Lemma 2.3. Suppose $1 \leq p_{0}<p_{1}<\infty$ and that $T$ is a sublinear operator of weak-type $p_{0}$ and $p_{1}$, with respect to the measure $\mu$, with norms $R_{0}$ and $R_{1}$ respectively, then $T$ is actually bounded on $L^{p}(d \mu)$ for all $p_{0}<p<p_{1}$. In fact, for any $0<t<1$,

$$
\|T f\|_{L^{p_{t}(d \mu)}} \leq C_{t} R_{0}^{1-t} R_{1}^{t}\|f\|_{L^{p_{t}(d \mu)}}
$$

where

$$
\frac{1}{p_{t}}=\frac{1-t}{p_{0}}+\frac{t}{p_{1}} \quad \text { and } \quad C_{t}^{p_{t}}=\frac{2^{p_{t}}}{p_{t}}\left(\frac{p_{1}}{p_{1}-p_{t}}+\frac{p_{0}}{p_{t}-p_{0}}\right) .
$$

We shall only need to apply this lemma where $p_{0}$ and $p_{1}$ are in some fixed interval $[1, S], t=\frac{1}{2}$ and $R_{1}, R_{2} \leq R$. In this case, writing $p=p_{1 / 2}$, we simply get the inequality

$$
\|T f\|_{L^{p}(d \mu)} \leq \frac{C R}{\left(p_{1}-p_{0}\right)^{1 / p}}\|f\|_{L^{p}(d \mu)}
$$

where $C$ depends only on $S$.

Lemma 2.4. If $f \in L^{p}(w)$ and $f_{Q_{k}} \geq \alpha>0$ for each of the disjoint cubes $\left\{Q_{k}\right\}$, then

$$
\sum_{k} w\left(Q_{k}\right) \leq K_{w}\left(\frac{\|f\|_{L^{p}(w)}}{\alpha}\right)^{p} .
$$

Proof. We can assume that $f(x) \geq 0$ and that $\|f\|_{L^{p}(w)}=1$, without loss of generality. Now,

$$
\begin{aligned}
\sum_{k} w\left(Q_{k}\right) & \leq \int \sum_{k} \frac{w\left(Q_{k}\right)}{\alpha\left|Q_{k}\right|} \chi_{Q_{k}} f \leq\left\|\sum_{k} \frac{w\left(Q_{k}\right)}{\alpha\left|Q_{k}\right|} \chi_{Q_{k}}\right\|_{L^{\prime}(\sigma)}\|f\|_{L^{p}(w)} \\
& \leq\left(\sum_{k} \frac{w\left(Q_{k}\right)^{p^{\prime}}}{\alpha^{p^{\prime}}\left|Q_{k}\right|^{p^{\prime}}} \sigma\left(Q_{k}\right)\right)^{1 / p^{\prime}} \leq \frac{K_{\sigma}^{1 / p^{\prime}}}{\alpha}\left(\sum_{k} w\left(Q_{k}\right)\right)^{1 / p^{\prime}},
\end{aligned}
$$

since $\sigma \in A_{p^{\prime}}$ and so,

$$
\sum_{k} w\left(Q_{k}\right) \leq \frac{K_{\sigma}^{p / p^{\prime}}}{\alpha^{p}}=\frac{K_{w}}{\alpha^{p}}
$$

Using Lemma 2.4, our first main theorem is now easy to state and prove.

Theorem 2.5. If $w \in A_{p}$, then $\|M f\|_{L^{p}(w)}^{p} \leq C K_{w}^{p^{\prime}}\|f\|_{L^{p}(w)}^{p}$. The power $K_{w}^{p^{\prime}}$ is best possible.

Proof. First, we show that for $1 \leq p<\infty$,

$$
w(\{M f>\alpha\}) \leq C K_{w}\left(\|f\|_{L^{p}(w)} / \alpha\right)^{p} .
$$

Without loss of generality, we assume that $f(x) \geq 0$ and that $\|f\|_{L^{p}(w)}=1$. Suppose that $M f(x)>\alpha>0$ so that $f_{Q_{k}} \geq \alpha$ for some cube $Q_{x}$ centered at $x$. Let $A_{r}=\{x:|x|<r, M f(x)>\alpha\}$. The Besicovich covering lemma tells us that $A_{r}$ can be covered by the union of $N_{n}$ collections of disjoint cubes, on each of which the mean value of $f$ is at least $\alpha$. Choose the collection $\left\{Q_{k}\right\}$, whose union has maximal $w$-measure. Thus, $w\left(A_{r}\right) \leq N_{n} w\left(\bigcup_{k} Q_{k}\right) \leq C K_{w} / \alpha^{p}$, by Lemma 2.4. Letting $r \rightarrow \infty$, we get (2.6). 
Suppose now that $p>1$. By Lemma 2.1, $w$ is also an $A_{p-\varepsilon}$ weight with comparable norm, where $\varepsilon \sim K_{w, p}^{1-p^{\prime}}$ and, trivially, $w$ is an $A_{p+\varepsilon}$ weight, with norm no larger than $K_{w, p}$. Applying the Marcinkiewicz interpolation theorem to the corresponding weak-type results at $p-\varepsilon$ and $p+\varepsilon$, we get the strong-type result we require with the indicated bound for the operator norm.

To see that the power $K_{w}^{p^{\prime}}$ is best possible, we give an example for $\mathbf{R}$ (a similar example works in $\mathbf{R}^{n}$ for any $\left.n\right)$. Let $w(x)=|x|^{(p-1)(1-\delta)}$, so that $K_{w} \sim 1 / \delta^{p-1}$ by Lemma 1.4. Now, $f(x)=|x|^{-1+\delta} \chi_{[0,1]} \in L^{p}(w)$. It is easy to see that $M f \geq f / \delta$ and so, $\|M f\|_{L^{p}(w)}^{p} /\|f\|_{L^{p}(w)}^{p} \geq C \delta^{-p} \sim K_{w}^{p^{\prime}}$.

Remark 2.7. The proof of Coifman and Fefferman [4], will also give the best possible exponent $K_{w}^{p^{\prime}}$, when the proof is examined closely, but some other proofs of the boundedness of $M$, e.g. [14], will not do so. The dependence in the weak- $(p, p)$ inequality (2.6) was found and shown to be best possible by Muckenhoupt [15].

Remark 2.8. It is easy to prove, using (2.6), that if $w \in A_{q}$ for some $q<p$, then $\|M f\|_{L^{p}(w)}^{p} \leq C K_{w, q}\|f\|_{L^{p}(w)}^{p}$, where $C=C_{p, q}$ now depends on $q$ as well as $p$ (and $C_{p, q}$ gets very large when $q$ is very close to $p$ ).

Theorem 2.5 neatly sews up the dependence for the Hardy-Littlewood maximal operator. The dependence for singular integral operators is not at all as easy to handle and, in fact, we shall not be able to find the best power of $K_{w}$. The best we can do is as follows, which actually takes care of the maximal singular integral operator $T^{*}$.

Theorem 2.9. If $w \in A_{p}$, then $\left\|T^{*} f\right\|_{L^{p}(w)}^{p} \leq C K_{w}^{p^{\prime}+p}\|f\|_{L^{p}(w)}^{p}$. The best power of $K_{w}$ in this inequality must lie in the interval $\left[\max \left(p, p^{\prime}\right), p^{\prime}+p\right]$.

Proof. The proof of the boundedness of $T^{*}$ on $L^{p}(w)$ for $w \in A_{p}$ given in [4] will give the required exponent, as long as we sharpen one of the inequalities used, namely the good- $\lambda$ inequality

$$
\left|\left\{x \in Q: T^{*} f>2 \alpha, M f<\gamma \alpha\right\}\right| \leq C \gamma|Q|,
$$

which holds for any cube $Q$ in the Whitney decomposition of $\left\{T^{*} f>\alpha\right\}$. We replace it by the sharp good- $\lambda$ inequality

$$
\left|\left\{x \in Q: T^{*} f>2 \alpha, M f<\gamma \alpha\right\}\right| \leq C e^{-c / \gamma}|Q|,
$$

for such cubes, which is proven in Lemma 2.13 below.

To see that the dependence is best possible, we give examples on $\mathbf{R}$ (similar examples can be found in $\mathbf{R}^{n}$ for $\left.n>1\right)$. Choose $w(x)=|x|^{(p-1)(1-\delta)}$ and $f(x)=|x|^{-1+\delta} \chi_{[0,1]}$, so that $\int f^{p} w=1 / \delta$. For $x>2, H f(x) \sim 1 / \delta x$ and so

$$
\int_{2}^{\infty}|H f|^{p} w \sim 1 / \delta^{p+1} \sim \delta^{-p}\|f\|_{L^{p}(w)}^{p} .
$$

Since $\delta^{-p} \sim K_{w}^{p^{\prime}}$ the best power must be at least $p^{\prime}$. Since the operator norm for $T: L^{p}(w) \rightarrow L^{p}(w)$ can be at least $C K_{w}^{p^{\prime} / p}$, the operator norm for $T: L^{p^{\prime}}(\sigma) \rightarrow L^{p^{\prime}}(\sigma)$ is also at least $C K_{w}^{p^{\prime} / p}=C K_{\sigma}$. Thus, the best power in our estimate must be at least $\max \left(p, p^{\prime}\right)$ (an explicit example is provided by 
$f(x)=x^{\delta} \chi_{[0,1]}(x)$ and $T=H$; it is easy to show that $\|H f\|_{L^{p}(w,[-1,0])} \geq$ $C K_{w}\|f\|_{L^{p}(w)}$.

We must now prove the sharp good- $\lambda$ inequality (2.10). The proof is a modification, using standard good- $\lambda$ techniques, of Hunt's main result in [12] which deals with the conjugate function on the unit circle. First we state an elementary lemma which is needed.

Lemma 2.11. Suppose $f \in L^{\infty}(Q)$ and that $T$ is an operator for which

$$
|\{x: T g(x)>\alpha\}| \leq\left(\frac{C p\|g\|_{p}}{\alpha}\right)^{p}
$$

for all $g \in L^{p}$ and sufficiently large $p$ and $\alpha, C$ being a constant independent of $p$. Then,

$$
|\{x: T f(x)>\alpha\}| \leq C e^{-\alpha / e\|f\|_{\infty}}|Q| .
$$

Proof. It suffices to prove this result for large $\alpha$, since the result is trivial otherwise. Let $K=\|f\|_{\infty}$. Since $f \in L^{\infty}(Q), f \in L^{p}(Q)$ for all $1<p<\infty$ and $\|f\|_{p} \leq|Q|^{1 / p} K$, and so

$$
|\{x: T f(x)>\alpha\}| \leq\left(\frac{C p \|\left. f\right|_{p}}{\alpha}\right)^{p} \leq C|Q|\left(\frac{p K}{\alpha}\right)^{p} .
$$

Letting $p=\alpha / e K$, we get the required result.

Remark 2.12. If $T$ is the maximal operator, a singular integral operator, or a maximal singular integral operator, then it satisfies the condition of the above lemma (see [16, p. 48]).

Lemma 2.13. Let $\Omega=\bigcup Q_{j}$ be the Whitney covering of $\left\{T^{*} f>\alpha\right\}$. Then

$$
\left|\left\{x \in Q_{j}: T^{*} f(x)>2 \alpha, M f(x) \leq \gamma \alpha\right\}\right| \leq C e^{-c / \gamma}\left|Q_{j}\right| .
$$

Proof. We can clearly assume that $M f\left(x_{0}\right)<\gamma \alpha$ for some $x_{0} \in Q_{j}$, and that $\gamma$ is small. We write $f=f_{1}+f_{2}$, where $f_{1}=f_{100 Q_{j}}$, and $f_{2}=f_{\mathbf{R}^{n} \backslash 100 Q_{j}}$. By standard estimation (as in the proof of Theorem III in [4]), we get that, for $x \in Q_{j}, T^{*} f_{2}(x) \leq \alpha+C \gamma \alpha<3 \alpha / 2$, if $\gamma$ is small enough.

To handle $f_{1}$, we first let $\Omega^{\prime}=\bigcup P_{k}$ be the Whitney decomposition of $\left\{M f_{1}>2^{n} \gamma \alpha\right\}$, where $n$ is the dimension. Note that $\left\|f_{1}\right\| \leq(101)^{n} \gamma \alpha\left|Q_{j}\right|$, and so $\Omega^{\prime} \subset 200 Q_{j}$. Let

$$
g(x)= \begin{cases}f_{1}(x), & x \notin \Omega^{\prime}, \\ \left(f_{1}\right)_{P_{k}}, & x \in P_{k},\end{cases}
$$

and $b=f_{1}-g$. Then $g$ is supported in $200 Q_{j},\|g\|_{\infty} \leq C \gamma \alpha$ and so, by Lemma 2.11,

$$
\left|\left\{x \in Q_{j}: T^{*} g>\alpha / 4\right\}\right| \leq C e^{-c / \gamma}\left|Q_{j}\right| .
$$

As for $b$, let us define $\Omega^{\prime \prime}=\bigcup 2 P_{k}$. Since $\int_{P_{k}} b=0$, we have, for $x \notin \Omega^{\prime \prime}$,

$$
T^{*} b(x) \leq \sum_{k} \int_{P_{k}}|b(t)|\left|K(x-t)-K\left(x-t_{k}\right)\right|,
$$


where $t_{k}$ is the centre of $P_{k}$. It follows that

$$
\begin{aligned}
T^{*} b(x) & \leq \sum_{k} \int_{P_{k}}|b(t)|\left(\frac{\delta_{k}}{\delta_{k}^{n+1}+\left(t_{k}-x\right)^{n+1}}\right) d t \\
& \leq C \gamma \alpha \sum_{k} \frac{\delta_{k}}{\delta_{k}^{n+1}+\left(t_{k}-x\right)^{n+1}} \equiv C \gamma \alpha \Delta(x)
\end{aligned}
$$

where $\delta_{k}$ is the diameter of $P_{k}$.

Carleson's [2] exponential estimate of $\Delta$ tells us that

$$
\left|\left\{x \in Q_{j}: \Delta(x)>c / \gamma\right\}\right| \leq C e^{-c / \gamma}\left|Q_{j}\right|
$$

and so, since $M f(x)>\gamma \alpha$ if $x \in \Omega^{\prime \prime}$,

$$
\left|\left\{x \in Q_{j}: T^{*} b(x)>\alpha / 4, M f(x) \leq \gamma \alpha\right\}\right| \leq C e^{-c / \gamma}\left|Q_{j}\right|
$$

This, together with our estimates for $f_{2}$ and $g$, is easily seen to imply the desired result.

Let us now examine $K_{w}$-dependence of operator norms for a particular class of weights, namely power weights. In the case of the Hardy-Littlewood maximal operator on power weighted spaces, we can clearly do no better than Theorem 2.5 (or Remark 2.8 for negative power weights, which are in $A_{1}$ ), since all our examples so far have involved power weights. However, in contrast to the case of general $A_{p}$ weights, we can also give a best possible dependence result for singular integral operators.

Theorem 2.14. If $T$ is a singular integral operator on $\mathbf{R}^{n}$ and $0<\delta<1$, then

(i) $w\left(\left\{T^{*} f>\alpha\right\}\right) \leq\left(C K_{w} / \alpha\right) \int|f| w$, if $w(x)=|x|^{-n(1-\delta)}$.

(ii) $\int\left|T^{*} f\right|^{p} w \leq C K_{w}^{p} \int|f|^{p} w$, if $w(x)=|x|^{-n(1-\delta)}$.

(iii) $\int\left|T^{*} f\right|^{p} w \leq C K_{w}^{p^{\prime}} \int|f|^{p} w$, if $w(x)=|x|^{n(p-1)(1-\delta)}$.

The exponents in (i)-(iii) are best possible.

Proof. We first prove (i). By normalization, we can assume that $\|f\|_{L^{1}(w)}=1$. We write $A_{j}=\left\{x \in \mathbf{R}^{n}: 2^{j} \leq|x|<2^{j+1}\right\}, f_{j}=f \chi_{A_{j}}, f_{j, 1}=f \chi_{\left\{|x|<2^{j+2}\right\}}$, and $f_{j, 2}=f-f_{j, 1}$. Clearly,

$$
\begin{aligned}
w\left(\left\{T^{*} f>\alpha\right\}\right) & =\sum_{j=-\infty}^{\infty} w\left(\left\{T^{*} f>\alpha\right\} \cap A_{j}\right) \\
& \leq \sum_{i=1}^{2} \sum_{j=-\infty}^{\infty} w\left(\left\{T^{*} f_{j, i}>\alpha / 2\right\} \cap A_{j}\right) \\
& =\sum_{I=1}^{2} S_{i}, \quad \text { say. }
\end{aligned}
$$


Now,

$$
\begin{aligned}
S_{1} & \leq \sum_{j=-\infty}^{\infty} 2^{-j n(1-\delta)}\left|\left\{T^{*} f_{j, 1}>\alpha / 2\right\} \cap A_{j}\right| \\
& \leq C\left(\sum_{j=-\infty}^{\infty} \frac{2^{-j n(1-\delta)}}{\alpha} \int\left|f_{j, 1}\right|\right), \quad \text { by the unweighted theory } \\
& =\frac{C}{\alpha} \sum_{j=-\infty}^{\infty} 2^{-j n(1-\delta)} \sum_{k \leq j+1} \int\left|f_{k}\right| \\
& =\frac{C}{\alpha} \sum_{k=-\infty}^{\infty} \int\left|f_{k}\right|\left(\sum_{j \geq k-1} 2^{-j n(1-\delta)}\right) \\
& \leq \frac{C}{\alpha} \sum_{k=-\infty}^{\infty} 2^{-n k(1-\delta)} \int\left|f_{n}\right| \leq \frac{C}{\alpha} .
\end{aligned}
$$

As for $S_{2}$, we note first that,

$$
\begin{aligned}
1 & =|f| w=\sum_{k} \int_{A_{k}}|f| w \geq C \sum_{k=-\infty}^{\infty} 2^{-k n(1-\delta)} \int_{A_{k}}|f| \\
& \geq C \sum_{k=-\infty}^{\infty} 2^{k n \delta} f_{A_{k}}|f|
\end{aligned}
$$

and so, if $x \in A_{j}$ then

$$
\begin{aligned}
T^{*} f_{j, 2}(x) & \leq \sum_{k>j+1} T^{*} f_{k}(x) \leq C\left(\sum_{k>j+1} \int_{A_{k}} \frac{|f(y)|}{|x-y|^{n}}\right) \\
& \leq C\left(\sum_{k>j+1} f_{A_{k}}|f|\right) \leq C 2^{-j n \delta} .
\end{aligned}
$$

But if $2^{-j n \delta}>c \alpha$, then

$$
j<\frac{-\log _{2} c \alpha}{n \delta} \equiv j_{0}
$$

It follows that

$$
S_{2} \leq \int_{|x|<2^{j_{0}}}|x|^{-n(1-\delta)} d x \sim \frac{2^{n \delta j_{0}}}{\delta} \sim \frac{1}{\alpha \delta} \sim \frac{K_{w}}{\alpha}
$$

We next prove (iii). Here $w(x)=|x|^{(p-1)(1-\delta)}$. We define $A_{j}$ as before, but now we define $f_{j, 1}=f \chi_{\left\{|x|<2^{j-1}\right\}}$, and $f_{j, 2}=f-f_{j, 1}$. Now, as in the $S_{1}$ case 
of (i),

$$
\begin{aligned}
\sum_{j=-\infty}^{\infty} \int_{A_{j}}\left|T^{*} f_{j, 2}\right|^{p} w & \leq C \sum_{j=-\infty}^{\infty} 2^{j n(p-1)(1-\delta)} \int_{A_{j}}\left|T^{*} f_{j, 2}\right|^{p} \\
& \leq C \sum_{j=-\infty}^{\infty} 2^{j n(p-1)(1-\delta)} \int\left|f_{j, 2}\right|^{p} \\
& =C \sum_{k=-\infty}^{\infty} \int\left|f_{k}\right|\left(\sum_{j \leq k+1} 2^{j n(p-1)(1-\delta)}\right) \\
& \leq C \sum_{k=-\infty}^{\infty} 2^{k n(p-1)(1-\delta)} \int\left|f_{n}\right| \\
& \leq C \int|f|^{p} w .
\end{aligned}
$$

As for the other terms, it is easy to see that if $x \in A_{j}$ then $T^{*} f_{j, 1} \leq$ $C M f_{j, 1} \leq C M f(x)$. Now using Theorem 2.5, we get

$$
\sum_{j=-\infty}^{\infty} \int_{A_{j}}\left|T^{*} f_{j, 1}\right|^{p} w \leq C \int|M f|^{p} w \leq C K_{w}^{p^{\prime}} \int|f|^{p} w .
$$

Part (iii) now follows readily from the estimates of the last two paragraphs.

We next prove (ii). To see this, let $w(x)=|x|^{n(p-1)(1-\delta)}$. By (iii), we have $\left\|T^{*} f\right\|_{L^{P}(w)} \leq C K_{w}^{p^{\prime} / p}\|f\|_{L^{p}(w)}$, and so by Lemma 1.5,

$$
\left\|T^{*} f\right\|_{L^{p^{\prime}(\sigma)}} \leq C K_{w}^{p^{\prime} / p}\|f\|_{L^{p^{\prime}}(\sigma)} \text {. }
$$

But $K_{w, p}^{p^{\prime} / p}=K_{\sigma, p^{\prime}}$ and $\sigma(x)=|x|^{-n(1-\delta)}$, giving us our required result on $L^{p^{\prime}}(\sigma)$.

We are left with giving examples to show that the exponents in (i)-(iii) are best possible. In (i), we let $f=\chi_{[1,2]}, T=H$, the Hilbert transform, and $\alpha=1 / 3$. Then $|H f(x)|>1 / 2$ for $x \in[0,1]$ and $\int_{0}^{1}|x|^{-1+\delta} d x=1 / \delta \sim K_{w}$. In (ii) and (iii), the examples given in the proof of Theorem 2.9 suffice.

We now turn our attention to the Marcinkiewicz integral operator $J_{\Omega}$, associated with an open set $\Omega$ of finite measure, which is defined for all $f: \Omega \rightarrow$ $[0, \infty)$ by the equation

$$
J_{\Omega}(f)(x)=\int_{\Omega} f(y) \frac{\delta(y)}{\delta(y)^{n+1}+|x-y|^{n+1}} d y,
$$

where $\delta(y)=\operatorname{dist}\left(y, \Omega^{c}\right)$. This is the version of the Marcinkiewicz integral operator used by Carleson in [2]. $J_{\Omega}$ is an important tool for controlling singular integral operators (see [16]). The following result summarizes the dependence of the resulting operator norm on the $A_{p}$ norm of $w$.

Thoerem 2.15. If $1 \leq p<\infty$, then $J_{\Omega}$ is bounded on $L^{p}(w)$ uniformly for all open $\Omega$ of finite measure if and only if $w \in A_{p}$. Furthermore, $\left\|J_{\Omega} f\right\|_{L^{p}\left(w, \mathbf{R}^{n}\right)} \leq$ $C K_{w}\|f\|_{L^{p}(w, \Omega)}$. The dependence on $K_{w}$ is best possible.

To prove Theorem 2.15, we first need the following lemma. 
Lemma 2.16. For any functions $f, g \geq 0$,

$$
\int_{\mathbf{R}^{n}}\left(J_{\Omega} f\right) g \leq C \int_{\Omega} f M g .
$$

Proof. By Fubini's theorem,

$$
\int_{\mathbf{R}^{n}}\left(J_{\Omega} f\right)(x) g(x) d x=\int_{\Omega} f(y) \delta(y)\left(\int_{\mathbf{R}^{n}} \frac{g(x)}{\delta(y)^{n+1}+|x-y|^{n+1}} d x\right) d y .
$$

Letting $\mathscr{A}_{k}=\left\{u \in \mathbf{R}^{n}: 2^{k} \delta(y) \leq|u| \leq 2^{k+1} \delta(y)\right\}$ and making the change of variable $u=x-y$, we get

$$
\begin{aligned}
\int_{\mathbf{R}^{n}} & \frac{g(x)}{\delta(y)^{n+1}+|x-y|^{n+1}} d x \\
& \leq \int_{|u| \leq \delta(y)} \frac{g(y+u) d u}{\delta(y)^{n+1}}+\sum_{k=0}^{\infty} \int_{\mathscr{A}_{k}} \frac{g(y+u) d u}{|u|^{n+1}} \\
& \leq \frac{C M g(y)}{\delta(y)}\left(1+\sum_{k=0}^{\infty} 2^{-k}\right) \leq \frac{C M g(y)}{\delta(y)}
\end{aligned}
$$

The required result now follows easily.

Proof of Theorem 2.15. Suppose $J_{\Omega}$ is bounded on $L^{p}(w)$ uniformly for all open $\Omega$ of finite measure. Fix a cube $Q$, and let $\Omega=2 Q$. Then, for any nonnegative function $f$ supported on $Q, J_{2 Q} f(x) \sim f_{Q}$ for all $x \in Q$. If $p>1$, let $f=\chi_{Q} w^{-1 /(p-1)}$. Because $J_{2 Q}$ is bounded on $L^{p}(w)$, it follows that

$$
\left(\int_{Q} w\right)\left(f_{Q} w^{-1 /(p-1)}\right)^{p} \leq C \int_{Q} w^{-1 /(p-1)}
$$

which clearly implies $w \in A_{p}$.

For the case $p=1$, let $f=\chi_{S}$ for an arbitrary measurable subset $S$ of $Q$. The boundedness of $J_{2 Q}$ on $L^{p}(w)$ now implies that

$$
\frac{|S|}{|Q|} w(Q) \leq C w(S)
$$

If we take $S=\{x \in Q: w(x)<a\}$ for any $a>a_{0} \equiv \operatorname{essinf}_{Q} w$, and then let $a \rightarrow a_{0}$, we get $w_{Q} \leq C a_{0}$, i.e. $w \in A_{1}$.

In proving the converse, we may assume, without loss of generality, that $f$ is supported on $\Omega$ and that $\|f\|_{L^{p}(w)}=1$. The case $p=1$ follows by letting $g=w$ in Lemma 2.16, so we assume $1<p<\infty$. If $g$ is a function for which $\|g\|_{L^{p^{\prime}(\sigma)}}=1$, then Lemma 2.16 tells us that,

$$
\begin{aligned}
\int_{\mathbf{R}^{n}}(J f) g & \leq C \int_{\Omega} f M g \\
& \leq C\left(\int_{\Omega} f^{p} w\right)^{1 / p}\left(\int_{\Omega}(M g)^{p^{\prime}} \sigma\right)^{1 / p^{\prime}} \\
& \leq C K_{\sigma, p^{\prime}}^{p / p^{\prime}}=C K_{w, p} .
\end{aligned}
$$

The required boundedness follows by duality. To see that this dependence is best possible, we let $w(x)=|x|^{-1+\delta}, f(x)=x^{\delta} \chi_{[0,1]}(x)$, and $\Omega=(0,1)$. 
Now for $0<x<1$, it is clear that

$$
J_{\Omega} f(-x)>\frac{1}{2} \int_{x}^{1} y^{\delta-1} d y=\frac{\left(1-x^{\delta}\right)}{2 \delta}
$$

and so,

$$
\begin{aligned}
\|J f\|_{L^{p}(w,[-1,0])}^{p} & >\frac{1}{2^{p} \delta^{p}} \int_{-1}^{0}\left(|x|^{-1+\delta}-p|x|^{-1+(p+1) \delta}\right) d x \\
& =\frac{1}{(p+1) 2^{p} \delta^{p+1}}
\end{aligned}
$$

whereas $\|f\|_{L^{p}(w)}^{p}=1 /(p+1) \delta$. Since $K_{w} \sim 1 / \delta$, it follows that

$$
\|J f\|_{L^{p}} /\|f\|_{L^{p}(w)} \geq C K_{w},
$$

as required.

Remark 2.17. Our three operator dependence results

$$
\begin{aligned}
\|M f\|_{L^{p}(w)}^{p} & \leq C K_{w}^{p^{\prime}}\|f\|_{L^{p}(w)}^{p}, \\
\|T f\|_{L^{p}(w)}^{p} & \leq C K_{w}^{p+p^{\prime}}\|f\|_{L^{p}(w)}^{p}, \\
\|J f\|_{L^{p}(w)}^{p} & \leq C K_{w}^{p}\|f\|_{L^{p}(w)}^{p}
\end{aligned}
$$

tie together well intuitively because, if $f$ is a function of bounded support $B$ then, roughly speaking, $J f$ can be as "nasty" as $T f$ near $B$, but tends to be smaller than it far from $B$, whereas $M f$ can control $T f$ far away from $B$, but not near $B$.

By way of contrast with the $K_{w}$-dependence of the above operators, let us finish by looking at simple averaging operators of the form $T_{Q}(f)=f * \chi_{Q} /|Q|$, where $Q$ is some cube. $T_{Q}$ is of course dominated by the maximal operator, which proves that for any $w \in A_{p}, T_{Q}$ is bounded on $L^{p}(w)$ (at least for $1<$ $p<\infty)$ with norm-dependence on $w$ of the form $K_{w}^{p^{\prime}}$. Intuitively, however, $T_{Q}$ is so "close" to the identity operator that we expect to be able to get a better exponent than $p^{\prime}$. The following lemma shows that this is indeed the case (simple examples show it is best possible).

Lemma 2.18. If $1 \leq p<\infty, T_{Q}$ is bounded on $L^{p}(w)$ uniformly for all cubes $Q$ if and only if $w \in A_{p}$. Furthermore, for any cube $Q$ centered at $0,\left\|T_{Q} f\right\|_{L^{p}(w)}^{p} \leq$ $C K_{w}\|f\|_{L^{p}(w)}^{p}$.

Proof. We may assume, without loss of generality, that $f \geq 0$. This allows us to also assume $Q$ is centered at 0 , since otherwise we can bound $T_{Q} f$ by a constant (dependent on $Q$ ) times $T_{Q^{\prime}} f$ where $Q^{\prime}$ is the smallest cube centered at 0 containing $Q$.

Divide $\mathbf{R}^{n}$ into the unique mesh $M$ of cubes of equal sidelength and disjoint interiors for which $Q \in M$. Suppose first that $f$ is supported in some $Q_{0} \in M$ and so $T_{Q} f$ is supported in $3 Q_{0}$. If $p>1$ then by Hölder's inequality,

$$
\begin{aligned}
\int_{3 Q_{0}}\left(\frac{\chi_{Q}}{|Q|} * f\right)^{p} w & \leq \frac{1}{|Q|^{p}} \int_{3 Q_{0}}\left(\int_{(Q+x) \cap Q_{0}} f^{p}(y) w(y) d y\right) \sigma\left(Q_{0}\right)^{p-1} w(x) d x \\
& =\frac{\sigma\left(Q_{0}\right)^{p-1}}{|Q|^{p}}\left(\int_{3 Q_{0}} w(x) d x\right) \int f^{p} w \leq C K_{w} \int f^{p} w,
\end{aligned}
$$


as required. In the case $p=1$, we simply estimate

$$
\begin{aligned}
& \frac{1}{|Q|} \int_{3 Q_{0}}\left(\int_{Q+x} f(y) d y\right) w(x) d x \\
& \quad \leq \frac{C}{|Q|}\left(\int f w\right)\left(\int_{3 Q_{0}} w(x) d x\right) / \underset{y \in Q_{0}}{\operatorname{essinf}} w(y) \\
& \quad \leq C K_{w}\left(\int f w\right) .
\end{aligned}
$$

For a general function $f$, we simply decompose $f=\sum_{C \in M} f \chi_{C}$, and we get the required result because of the limited amount of overlap among the supports of the functions $\left.\left\{\chi_{Q} /|Q| * f \chi_{C}\right)\right\}_{C \in M}$.

\section{REVERSE JENSEN INEQUALITIES}

In this section, we examine some rather general reverse Jensen inequalities and show their connection to the conditions $A_{p}(d \mu)$ and $R H_{p}(d \mu)$. The $R H_{p}(d \mu)$ condition (defined below) was first examined by Gehring [10] (in the case $\mu=$ Lebesgue measure), and it was Coifman and C. Fefferman [4] who first showed the close relation between $R H_{p}$ and $A_{p}$ conditions (they showed that a weight is in some $A_{p}$ space if and only if it is in some $R H_{q}$ space, but there is no possible relationship between $p$ and $q$ ).

Since then, the $R H_{p}$ condition has become important in its own right in the theory of elliptic operators on Lipschitz spaces. Dahlberg [5] showed that the Dirichlet problem for such operators is solvable with $L^{p}$ boundary values if and only if harmonic measure is in $R H_{p}(d \sigma)$, where $d \sigma$ is surface measure. For further results in this direction, see [6, 7 and 8].

We say a positive Borel measure $\mu$ is a doubling measure, $\mu \in \mathbf{D}$, if $\mu(2 Q) \leq$ $C \mu(Q)$ for all cubes $Q$. We say $w$ is a doubling weight if $w d x$ is a doubling measure. If $Q$ is a cube we denote by $l(Q)$ the sidelength of $Q$. We define $\log ^{+} x \equiv \log (2+x)$.

Definition. If $\mu \in \mathbf{D}$ and $1<p<\infty$, we say that $w$ is a $R H_{p}(d \mu)$ weight if

$$
\left(f_{Q} w^{p} d \mu\right)^{1 / p} \leq K f_{Q} w d \mu
$$

for all cubes $Q$. The smallest such $K$ is referred to as the $R H_{p}(d \mu)$-norm of $w$.

Condition (3.1) is often called a "reverse Hölder inequality," because it is Hölder's inequality with the direction of the inequality reversed (Hölder's inequality is of course true with $K=1$ ). More generally, if $\|\cdot\|_{1, Q}$ and $\|\cdot\|_{2, Q}$ are norms for functions defined on an arbitrary cube $Q$, and Jensen's inequality implies that $\|f\|_{1, Q} \leq C_{1}\|f\|_{2, Q}$, then we refer to the condition $\|w\|_{2, Q} \leq C_{2}\|w\|_{1, Q}$ as a reverse Jensen inequality (we will only be interested in such inequalities when they hold uniformly for all cubes $Q$ ).

If $\mu_{1}$ and $\mu_{2}$ are positive doubling measures, we say that $\mu_{1}$ is comparable to $\mu_{2}$ if there exist $\alpha, \beta \in(0,1)$ such that $\mu_{1}(E) / \mu_{1}(Q)<\beta$ whenever $\mu_{2}(E) / \mu_{2}(Q)<\alpha$ for every $E \subseteq Q$, and every cube $Q$. Let us now state a result taken directly from [4], which is very useful for our purposes. 
Lemma 3.2. If $\mu_{1}$ and $\mu_{2}$ are positive doubling measures, the following are equivalent:

(i) There exists $C, \delta>0$ such that for every $E \subseteq Q \subset \mathbf{R}^{n}$,

$$
\frac{\mu_{2}(E)}{\mu_{2}(Q)} \leq C\left(\frac{\mu_{1}(E)}{\mu_{1}(Q)}\right)^{\delta} .
$$

(ii) $\mu_{2}$ is comparable to $\mu_{1}$.

(iii) $\mu_{1}$ is comparable to $\mu_{2}$.

(iv) $d \mu_{2}=w(x) d \mu_{1}(x)$ and for every cube $Q$,

$$
\left(f_{Q} w^{1+\delta} d \mu_{1}\right)^{\frac{1}{1+\delta}} \leq C f_{Q} w d \mu_{1} .
$$

Lemma 3.2 allows us to prove the following lemma, which generalizes to $A_{p}(d \mu)$ and $R H_{p}(d \mu)$, results which are well known for $\mu=$ Lebesgue measure. For the rest of the section, $\mu$ is an arbitrary but fixed doubling measure on $\mathbf{R}^{n}$, and $d \nu=w d \mu$.

Lemma 3.3. If $w \in A_{\infty}(d \mu)$ then $\nu \in \mathbf{D}$. Furthermore,

$$
A_{\infty}(d \mu)=\bigcup_{1<p<\infty} R H_{p}(d \mu)=\bigcup_{1<q<\infty} A_{q}(d \mu) .
$$

Proof. If we prove that $\nu \in \mathbf{D}$, the rest of the lemma follows fairly easily from Lemma 3.2 (alternatively, it is implied by Theorem 15 in Chapter 1 of Strömberg and Torchinsky [17]), so we shall confine ourselves to proving that $\nu \in \mathbf{D}$.

First note that the condition $\mu \in \mathbf{D}$ is equivalent to assuming there is some $C_{1}>0$ for which

$$
\mu\left(Q^{\prime}\right) \leq C_{1} \mu(Q)
$$

for all cubes $Q, Q^{\prime}$ which are adjacent and of equal size.

We will now show, roughly speaking, that a very thin slice from a side of a cube has very small $\mu$-measure compared with the full cube. For simplicity, we will prove this for the cube $Q_{0}=\left\{x:\left|x_{i}\right| \leq 1\right\}$ and the slice $S_{\varepsilon} \equiv\left\{x:\left|x_{i}\right| \leq\right.$ $\left.1, x_{1}>1-\varepsilon\right\}$

We divide $Q_{0}$ into $2^{n}$ cubes of sidelength 1 , half of which are in the slice $S_{1}$. Applying the estimate (3.4) to each subcube in $S_{1}$ and its adjacent subcube in $Q_{0} \backslash S_{1}$, gives us the inequality $\mu\left(S_{1}\right) \leq\left(C_{1} /\left(C_{1}+1\right)\right) \mu\left(Q_{0}\right)$. This process can be continued to give $\mu\left(S_{2-(k+1)}\right) \leq\left(C_{1} /\left(C_{1}+1\right)\right) \mu\left(S_{2-k}\right)$ (to see this, simply divide $S_{2-k}$ into $2^{k n+n-k}$ cubes of sidelength $2^{-(k+1)}$, half of which are in $S_{2-(k+1)}$, and half in $\left.S_{2-k} \backslash S_{2-(k+1)}\right)$. Thus

$$
\mu\left(S_{2-k}\right) \leq\left(C_{1} /\left(C_{1}+1\right)\right)^{k+1} \mu\left(Q_{0}\right),
$$

and so

$$
\mu\left(S_{\varepsilon}\right) / \mu(Q) \rightarrow 0 \quad(\varepsilon \rightarrow 0) .
$$

Clearly, the above argument will work equally well if we let $Q_{0}$ be an arbitrary cube and $S_{\varepsilon}$ be a slice of thickness $l(Q) / 2 \varepsilon$ and, in fact, the convergence in (3.5) is uniform for all such cubes and slices. 
We are now ready to show that $\nu \in \mathbf{D}$. Given a cube $Q_{0}$, let us write $Q_{\varepsilon} \equiv(1+\varepsilon) Q_{0}$ for any $\varepsilon>0$. Since one can get $Q_{0}$ from $Q_{\varepsilon}$ removing $2 n$ slices of thickness $\varepsilon l(Q)$, it follows that

$$
\frac{\mu\left(Q_{\varepsilon} \backslash Q_{0}\right)}{\mu\left(Q_{\varepsilon}\right)} \rightarrow 0 \quad(\varepsilon \rightarrow 0)
$$

uniformly for all cubes $Q_{0}$. Using the fact that $\nu \in A_{\infty}(d \mu)$ we conclude that $\nu\left(Q_{\varepsilon} \backslash Q_{0}\right) / \nu\left(Q_{\varepsilon}\right)<\frac{1}{2}$ for some sufficiently small $\varepsilon>0$. Thus $\nu\left(Q_{\varepsilon}\right)<2 \nu\left(Q_{0}\right)$ which we can iterate to get the doubling condition $\nu\left(Q_{1}\right)<2^{k} \nu\left(Q_{0}\right)$ for any $k>\log _{1+\varepsilon}(2)$.

Given exponents $0<q<p<\infty$, it is natural to consider the more general reverse Hölder's inequality

$$
\left(f_{Q} w^{p} d \mu\right)^{1 / p} \leq K\left(f_{Q} w^{q} d \mu\right)^{1 / q}
$$

Let us denote by $R H_{p, q}(d \mu)$ the class of weights satisfying (3.6) for all cubes $Q$. In fact we have not introduced anything new: if $p>1$ then $R H_{p, q}(d \mu)=$ $R H_{p}(d \mu)$ for any $0<q<p$. This follows as a special case of the "selfimproving" nature of these weights: if $w \in R H_{p, q}(d \mu)$, then $w \in R H_{p, r}(d \mu)$ for any $0<r<q$. To see this, we use both reverse and normal Hölder inequalities to get

$$
\begin{aligned}
\left(f_{Q} w^{p} d \mu\right)^{q / p} & \leq K\left(f_{Q} w^{q} d \mu\right) \\
& \leq K\left(f_{Q} w^{p} d \mu\right)^{\frac{q-r}{p-r}}\left(f_{Q} w^{r} d \mu\right)^{\frac{p-q}{p-r}}
\end{aligned}
$$

which clearly implies that $w \in R H_{p, r}(d \mu)$.

It is reasonable to extend the definition of $R H_{p}(d \mu)$ so that it is defined for all $p>0$ by the equation $R H_{p}(d \mu) \equiv R H_{p, q}(d \mu)$ for any $q<p$. The next lemma, which links $R H_{p}(d \mu)$ with $A_{\infty}(d \mu)$, is now easy to prove (this lemma is also to be found in Stromberg and Torchinsky [17]).

Lemma 3.7. If $0<p<\infty$, then $w \in R H_{p}(d \mu)$ if and only if $w^{p} \in A_{\infty}(d \mu)$.

Proof. If $w^{p} \in A_{\infty}(d \mu)$ then, by Lemma 3.3, $w^{p} \in R H_{q}(d \mu)$ for some $q>1$. Thus $w \in R H_{p q}(d \mu) \subset R H_{p}(d \mu)$.

For the converse, we may assume $p=1$. If $w \in R H_{1}(d \mu)$ then, for any $0<q<1, w^{q} \in R H_{1 / q}(d \mu)$ and so $w^{q} \in A_{p}(d \mu)$ for some $1<p<\infty$ by Lemma 3.3. It follows that

$$
\begin{aligned}
& \left(f_{Q} w d \mu\right)^{q}\left(f_{Q} w^{\frac{-q}{p-1}} d \mu\right)^{p-1} \\
& \quad \leq C\left(f_{Q} w^{q} d \mu\right)\left(f_{Q} w^{\frac{-q}{p-1}} d \mu\right)^{p-1} \leq C .
\end{aligned}
$$

The inequality between the first and last terms is essentially the defining inequality for $w \in A_{(p-1+q) / q}(d \mu)$, and so $w \in A_{\infty}(d \mu)$. 
Remark 3.8. We showed at the beginning of this proof that if $w \in R H_{p}(d \mu)$, then $w \in R H_{p+\varepsilon}(d \mu)$ for some $\varepsilon>0$. This analog for $R H_{p}$ of Lemma 2.1 was first proved by Gehring [10] in the case $\mu=$ Lebesgue measure.

The following lemma gives a couple of useful alternative characterizations of $A_{\infty}(d \mu)$ (the first of which is a reverse Jensen inequality). Part (i), for $\mu=$ Lebesgue measure, is due to Garcia-Cuerva and Rubio de Francia [9], and part (ii) is due to Coifman and C. Fefferman [4].

Lemma 3.9. Each of the following is equivalent to $w \in A_{\infty}(d \mu)$.

(i) For all cubes $Q$,

$$
f_{Q} w d \mu \leq C \exp \left(f_{Q} \log w d \mu\right) .
$$

(ii) There are constants $\alpha$ and $\beta$ such that for all cubes $Q$,

$$
\mu(\{x \in Q: w(x) \geq \beta \nu(Q) / \mu(Q)\})>\alpha \mu(Q) .
$$

Proof. We prove only (i), as the easy proof of (ii) for Lebesgue measure in [4] can be readily modified to handle the more general case. To prove (i), suppose that $w \in A_{\infty}(d \mu)$. Then

$$
f_{Q} w d \mu \leq C\left(f_{Q} w^{-\varepsilon} d \mu\right)^{-1 / \varepsilon} \leq C \exp \left(f_{Q} \log w d \mu\right)
$$

where the first inequality is because $w \in A_{q}(d \mu)$ for some $1<q$, and the second inequality is by Jensen's lemma (since $\log x^{-1 / \varepsilon}$ is convex).

Conversely, if (i) is satisfied, then we can apply Jensen's inequality with respect to the convex function $e^{x / 2}$ to get

$$
f_{Q} w d \mu \leq C \exp \left(f_{Q} \log w d \mu\right) \leq C\left(f_{Q} w^{1 / 2} d \mu\right)^{2}
$$

which implies $w \in A_{\infty}(d \mu)$.

We shall now examine more general reverse Jensen inequalities, but first we need to introduce some notation. Let $F$ be the class of continuous increasing functions mapping $[0, \infty)$ onto itself. If $\phi \in F$, we define

$$
\|f\|_{\phi\left(L_{Q}\right)(d \mu)}=\inf \left\{C>0: f_{Q} \phi\left(\frac{|f(x)|}{C}\right) d \mu(x) \leq 1\right\}
$$

if it exists. If $\phi$ is convex, this is the usual Orlicz norm with respect to $Q$ normalized Lebesgue measure. In other cases, this "norm" can still be defined but it does not satisfy the triangle inequality.

If $\phi_{1}, \phi_{2} \in F, \phi_{2} \circ \phi_{1}^{-1}$ is convex, and $\phi_{2}(2 x) / \phi_{2}(x)>1+\varepsilon_{2}$, then it follows from Jensen's inequality that, for all cubes $Q,\|f\|_{\phi_{1}\left(L_{Q}\right)(d \mu)} \leq C\|f\|_{\phi_{2}\left(L_{Q}\right)(d \mu)}$, $C$ being a constant that depends only on $\phi_{2} \circ \phi_{1}^{-1}(1)$ and $\varepsilon_{2}$ (the $\varepsilon_{2}$ condition is unnecessary if $\left.\phi_{2} \circ \phi_{1}^{-1}(1) \geq 1\right)$. We are interested in the connection between conditions involving $A_{p}(d \mu)$ or $R H_{p}(d \mu)$ and inequalities of reverse Jensen type which hold uniformly for all cubes, i.e. inequalities of the form

$$
\|w\|_{\phi_{2}\left(L_{Q}\right)(d \mu)} \leq C_{0}\|w\|_{\phi_{1}\left(L_{Q}\right)(d \mu)} \text { for all cubes } Q
$$


where $w$ is some weight, $C_{0}$ is some constant and $\phi_{2} \circ \phi_{1}^{-1}$ is convex (or satisfies some related condition). For example, if $\phi_{2}(x)=x^{2}$, and $\phi_{1}(x)=x$, then (3.11) is the defining condition for $w \in R H_{2}(d \mu)$.

We are mainly interested in functions which "grow like powers of $x$ " (as opposed to exponentially, or logarithmically, or other such growth), so we will make assumptions such as $\phi_{i}(2 x) \leq C_{i} \phi_{i}(x)$ or $\phi_{i}(2 x) \geq\left(1+\varepsilon_{i}\right) \phi_{i}(x)$ whenever they are useful for our purposes.

If there is some $c>0$ for which

$$
\phi_{1}(x)>\phi_{2}(c x) \text { for all } x>0,
$$

then (3.11) is trivially true, so we confine our interest to the case where

$$
\phi_{1}(x) / \phi_{2}(c x) \rightarrow 0(x \rightarrow \infty) \text { for all } c>0 .
$$

This is not a very restrictive assumption because, if $\phi_{2} \circ \phi_{1}^{-1}$ is convex and if $\left(1+\varepsilon_{2}\right) \phi_{2}(x) \leq \phi_{2}(2 x) \leq C_{2} \phi_{2}(x)$ (in which case (3.13) can be written simply as $\left.\phi_{1}(x) / \phi_{2}(x) \rightarrow 0(x \rightarrow \infty)\right)$, it is easily seen that (3.13) is true whenever (3.12) is false. Interestingly, (3.13) makes superfluous the assumption that $\phi_{2} \circ \phi_{1}^{-1}$ is convex. In fact, our first result is the following.

Proposition 3.14. If $\phi_{1}$ is convex, and (3.13) and (3.11) are both satisfied, then $w \in A_{\infty}(d \mu)$.

Proof. Suppose that $w \notin A_{\infty}(d \mu)$. Let us fix $0<\varepsilon<1 / 4$ and let $m$ be so large that $\phi_{1}(x) / \phi_{2}\left(x / C_{0}\right)<\varepsilon$ whenever $x>\phi_{1}^{-1}(m / 4)$. Then, by Lemma 3.9 , there is a cube $Q$ for which $\mu(S)>(1-1 / m) \mu(Q)$, where

$$
S=\left\{x \in Q: w(x) \leq \frac{\phi_{1}^{-1}(1 / 2)}{\phi_{1}^{-1}(1)} f_{Q} w d \mu\right\} .
$$

Letting $\hat{w}=w /\|w\|_{\phi_{1}\left(L_{Q}\right)(d \mu)}$, we see that $f_{Q} \hat{w} d \mu \leq \phi_{1}^{-1}(1)$ by Jensen's lemma. Thus, if $x \in S, \phi_{1}(\hat{w}(x)) \leq 1 / 2$ and so $\int_{Q \backslash S} \phi_{1} \circ \hat{w} \geq 1 / 2$. Since $\mu(Q \backslash S) \leq$ $\mu(Q) / m$, it follows that

$$
f_{L} \phi_{1} \circ \hat{w} d \mu \geq \frac{1}{4}
$$

where $L=\left\{x \in Q: \phi_{1}(\hat{w}(x))>m / 4\right\}$. From our definition of $m$, we get

$$
f_{Q} \phi_{2}\left(\frac{\hat{w}}{C_{0}}\right) d \mu>\frac{1}{4 \varepsilon}>1 \text {. }
$$

This contradicts (3.11), and so $w \in A_{\infty}(d \mu)$, as required.

As an example of this proposition, the case $\phi_{1}(x)=x, \phi_{2}(x)=x \log ^{+} x, \mu=$ Lebesgue measure, is to be found in [7]. Proposition 3.14 says that weaker conditions, such as that given by $\phi_{1}(x)=x, \phi_{2}(x)=x \log ^{+} \log ^{+} x$ are also sufficient to guarantee $x \in A_{\infty}(d \mu)$.

Ideally, we would like to generalize Lemma 3.7 by eliminating the hypothesis that $\phi_{1}$ is convex from the above theorem and proving that, assuming (3.13), $\phi_{2} \circ w \in A_{\infty}(d \mu)$ if and only if (3.11) holds. Unfortunately, this is not true. For example, let $\mu=$ Lebesgue measure, $w(x)=\min \left(1, x^{-1 / 2}\right), \phi_{1}(x)=x^{1 / 2}$ and

$$
\phi_{2}(x)= \begin{cases}x, & x \geq 10^{-10}, \\ 10^{10} x^{2}, & x<10^{-10}\end{cases}
$$


Then (3.11) is true, but $\phi_{2}(w(x))$ behaves like $\frac{1}{x}$ for large $x$, and so it is not an $A_{\infty}$ function.

Upon reflection, this counterexample reveals why we cannot prove such a result. If $\phi(x)$ is very small for $x<x_{0}$, the exact values of $\phi(x)$ for $x<x_{0}$ have very little effect on the $\phi\left(L_{Q}\right)$-norm of a function, whereas the $A_{\infty}(d \mu)$ condition is very much dependent on the relative size of the weight at different points, but independent of the average value of the weight in the interval. Also, whatever result we can get should reflect the invariance of reverse Jensen inequalities (involving a weight $w)$ under the transformations $w \mapsto b w(b>0)$ and the invariance of the condition $\phi \circ w \in A_{\infty}(d \mu)$ under the transformations $\phi \mapsto r \phi(r>0)$. The next theorem is fairly close to the result we want; it has the advantage of being true, but the disadvantage of involving a whole family of reverse Hölder inequalities, and thus being a more difficult condition to verify.

Theorem 3.15. If $\phi_{1}, \phi_{2} \in F, \phi_{1}(x) / x \rightarrow 0(x \rightarrow \infty)$, and $\left(1+\varepsilon_{2}\right) \phi_{2}(x) \leq$ $\phi_{2}(2 x) \leq C_{2} \phi_{2}(x)$, then the following are equivalent:

(i) $\|w\|_{r \phi_{2}\left(L_{Q}\right)(d \mu)} \leq C_{0}\|w\|_{\left(\phi_{1} \circ\left(r \phi_{2}\right)\right)\left(L_{Q}\right)(d \mu)}$, for all $r>0$, and all cubes $Q$.

(ii) $\phi_{2}(b w) \in A_{\infty}$ for every $b>0$.

Proof. Suppose (i) is true, but, for some fixed $b>0, w_{2} \equiv \phi_{2}(b w) \notin A_{\infty}(d \mu)$. We may assume $C_{0} \geq 1$ without loss of generality. Let us fix $\varepsilon>0$ and choose $m$ so large that $\phi_{1}(x) / x<\varepsilon$ whenever $x>\phi_{1}^{-1}(m / 4)$.

Since $w_{2} \notin A_{\infty}$, there is a cube $Q$ for which $\mu(S)>(1-1 / m) \mu(Q)$, where

$$
S=\left\{x \in Q: w_{2}(x) \leq \frac{\phi_{1}^{-1}(1 / 2)}{K} f_{Q} w_{2} d \mu\right\}
$$

and $K=C_{2}^{\log _{2}\left(C_{0}\right)+1}$. We choose $r$ so that $\int_{Q} \phi_{1}\left(r w_{2}\right) d \mu=\mu(Q)$. It follows from our hypotheses that $\int_{Q} r w_{2} d \mu \leq K \mu(Q)$ and so, for all $x \in S$, $\phi_{1}\left(r w_{2}(x)\right) \leq 1 / 2$. Arguing as in Proposition 3.14, we get

$$
f_{Q} r w_{2} d \mu>\frac{1}{4 \varepsilon}
$$

which is a contradiction for $\varepsilon \leq \frac{1}{4} K$.

Conversely, if (ii) is true, we show that (i) is true for fixed but arbitrary $r>0$. Since (ii) is true for all $b>0$, we can assume $\|w\|_{\left(\phi_{1} \circ r \phi_{2}\right)\left(L_{Q}\right)(d \mu)}=1$, without loss of generality. But now, by Lemma 3.9, we get that

$$
\mu\left(\left\{x \in Q: r \phi_{2}(w(x))>\beta\left(r \phi_{2} \circ w\right)_{Q}\right\}\right)>\alpha \mu(Q)
$$

for some $\alpha$ and $\beta$. It follows that $f_{Q} r \phi_{2}(w) d \mu \leq \frac{1}{\beta} \phi_{1}^{-1}(2 / \alpha)$ because, if this were not so, then

$$
\mu\left(\left\{x \in Q: \phi_{1}\left(r \phi_{2}(w(x))\right)>2 / \alpha\right\}\right)>\alpha \mu(Q),
$$

which contradicts the assumption $\|w\|_{\phi_{1} \circ \phi_{2}\left(L_{Q}\right)(d \mu)}=1$. It now follows from the " $1+\varepsilon_{2}$ " rate of growth assumption on $\phi_{2}$ that $\|w\|_{r \phi_{2}\left(L_{Q}\right)(d \mu)}$ is bounded, as required.

In the case $\phi_{2}(x)=x$, the parameters $r$ and $b$ in Theorem 3.15 become superfluous, and so we get the following corollary. 
Corollary 3.16. If $\phi_{1} \in F, \phi_{1}(x) / x \rightarrow 0(x \rightarrow \infty)$, then $w \in A_{\infty} \Leftrightarrow\|w\|_{L_{Q}^{1}(d \mu)} \leq$ $C_{0}\|w\|_{\phi_{1}\left(L_{Q}\right)(d \mu)}$.

Let us now look at a class of inequalities that generalize the definitions of $A_{p}(d \mu)$ and $R H_{p}(d \mu)$. We will replace the function $x \mapsto x^{p}$ by a whole class of similar functions, and associate a "norm" with each of these functions. We then define a partial ordering on these functions which has the property that if one function precedes another, its associated norm dominates the other; furthermore, for a particular weight $w$, there is a reverse inequality between these norms of $w$ holding uniformly for all cubes if and only if a particular power of $w$ is in $A_{p}(d \mu)$ for a particular $p(1<p \leq \infty)$.

We first define the class of functions $G_{1} \subset F$. If $\phi \in F$ then $\phi \in G_{1}$ if there are constants $a, \varepsilon>0$ for which:

(a) $a x \leq \phi(x)$,

(b) $\phi(x) / x^{r} \rightarrow 0(x \rightarrow \infty)$ for all $r>1$,

(c) $\phi(2 x) \geq(1+\varepsilon) \phi(x)$.

For example, the functions $x \mapsto x\left(\log ^{+} x\right)^{r}$, and $x \mapsto x\left(\log ^{+} \log ^{+} x\right)^{r}$ are in $G_{1}$ for any $r \geq 0$. We then define

$$
\begin{aligned}
& G_{p}=\left\{x \mapsto \phi\left(x^{p}\right): \phi \in G_{1}\right\}, \quad \text { for all } p \neq 0, \\
& G_{0}=\{\log \}, \quad G=\bigcup_{p \in \mathbf{R}} G_{p} .
\end{aligned}
$$

If $\phi \in G_{p}$, then $\phi$ has domain $[0, \infty)$ if $p>0$, and $\phi$ has domain $(0, \infty)$ if $p \leq 0$.

Suppose $\phi \in G_{p}$. If $p \neq 0$, and so $\phi(x)=\phi_{1}\left(x^{p}\right)$ for some $\phi_{1} \in G_{1}$, we define $\|w\|_{\phi\left(L_{Q}\right)(d \mu)}$ to mean $\left\|w^{p}\right\|_{\phi_{1}\left(L_{Q}\right)(d \mu)}^{1 / p}$, where the latter norm is previously defined because $\phi_{1} \in F$. If $\phi \in F$, then this definition of an $\phi$-norm is consistent with the previous definition. We define $\|w\|_{\log L_{Q}(d \mu)}$ in the obvious way, namely

$$
\|w\|_{\log L_{Q}(d \mu)} \equiv \exp \left(f_{Q} \log w d \mu\right) .
$$

We also define a partial ordering $\prec$ on $G$. Let $\phi_{i} \in G_{p_{i}}$ for $i=1,2$. Then

(i) For $p_{1}, p_{2}>0, \phi_{1} \prec \phi_{2}$ whenever $\phi_{1}(x) / \phi_{2}(x) \rightarrow 0(x \rightarrow \infty)$.

(ii) For $p_{1}, p_{2}<0, \phi_{1} \prec \phi_{2}$ whenever $\phi_{2}(x) / \phi_{1}(x) \rightarrow 0(x \rightarrow 0)$.

(iii) For $p_{1}<0<p_{2}, \phi_{1} \prec \log \prec \phi_{2}$.

In particular, it follows from the above and properties (a) and (b) of $G_{1}$ that if $p_{1}<p_{2}$ then $\phi_{1} \prec \phi_{2}$. Also, $x \mapsto x^{p}$ is a minimal element in $G_{p}$ for all $p>0$ and a maximal element in $G_{p}$ for all $p<0$. The next lemma shows that this partial ordering is indeed very natural for our purposes.

Lemma 3.17. If $\phi_{1}, \phi_{2} \in G$ and $\phi_{1} \prec \phi_{2}$ then

$$
\|w\|_{\phi_{1}\left(L_{Q}\right)(d \mu)} \leq C\|w\|_{\phi_{2}\left(L_{Q}\right)(d \mu)} \text {. }
$$

Proof. Suppose $\phi_{i} \in G_{p_{i}}$ for $i=1,2$. If $p_{1}>0$, then $\phi_{1}, \phi_{2} \in F$, and so the $\phi_{i}$-norm is as defined for $\phi \in F$. Now, since $\phi_{1} \prec \phi_{2}$, it follows that $\phi_{1}(x)<C \phi_{2}(x)$ for all $x \geq \phi_{1}^{-1}(1 / 2)$. Letting $\hat{w}=w /\|w\|_{\phi_{1}\left(L_{Q}\right)(d \mu)}$ and $L=\left\{x \in Q: \phi_{1}(\hat{w})>1 / 2\right\}$, it follows that

$$
f_{Q} \phi_{2}(\hat{w}) d \mu>\frac{1}{C} f_{L} \phi_{1}(\hat{w}) d \mu \geq \frac{1}{2 C} .
$$


Since property (c) of $G_{1}$ clearly extends to $G_{p}$ for all $p>0$, the desired conclusion follows easily.

If $p_{2}<0$, we can reduce to the first case by letting $\tilde{\phi}_{i}(x)=\phi_{i}(1 / x)$, because $\tilde{\phi}_{i} \in G_{-p_{i}}, \tilde{\phi}_{2} \prec \tilde{\phi}_{1}$, and

$$
\|w\|_{\phi_{i}\left(L_{Q}\right)(d \mu)}=\|1 / w\|_{\tilde{\phi}_{i}\left(L_{Q}\right)(d \mu)}^{-1} .
$$

If $p_{1}=0$ (so $\phi_{1}=\log$ ), then we can choose $p$ such that $0<p<p_{2}$, and it follows from Jensen's inequality, and the previously handled " $0<p_{1}<p_{2}$ " case, that

$$
\|w\|_{\log \left(L_{Q}\right)(d \mu)} \leq\|w\|_{L_{Q}^{p}(d \mu)} \leq C\|w\|_{\phi_{2}\left(L_{Q}\right)(d \mu)} .
$$

We can reduce the case $p_{2}=0$ to the case $p_{1}=0$ by taking reciprocal functions $\tilde{\phi}_{i}(x)$, as before. Finally, the case $p_{1}<0<p_{2}$ follows by combining the last two cases.

We are now ready to state and prove the main theorem which classifies all "reverse Jensen" inequalities involving functions in $G$.

Theorem 3.18. Suppose $\phi_{i} \in G_{p_{i}}$ for $i=1,2$, and $\phi_{1} \prec \phi_{2}$. Then, the inequality

$$
\|w\|_{\phi_{2}\left(L_{Q}\right)(d \mu)} \leq C\|w\|_{\phi_{1}\left(L_{Q}\right)(d \mu)} \text { for all cubes } Q
$$

is equivalent to

(i) $w^{p_{2}} \in A_{\infty}(d \mu)$, if $p_{1} \geq 0$ (equivalently, $w \in R H_{p_{2}}(d \mu)$ ).

(ii) $w^{p_{2}} \in A_{r}(d \mu)$, if $p_{2}>0>p_{1}$ (where $\left.r=\left(p_{1}-p_{2}\right) / p_{1}\right)$.

(iii) $w^{p_{1}} \in A_{\infty}(d \mu)$, if $p_{2} \leq 0$ (equivalently, $w^{-1} \in R H_{-p_{1}}(d \mu)$ ).

Proof. Let us first prove (i). It is sufficient to prove it in the case $p_{2}=1$, because of the way we defined $\|w\|_{\phi\left(L_{Q}\right)(d \mu)}$ for $\phi \in G_{p}$ for $p \neq 0$. Suppose $w$ satisfies (3.19). If $p_{1}<1$, then by property (a) of $G_{1}$ we see that

$$
\|w\|_{L_{Q}^{1}} \leq C\|w\|_{\phi_{2}\left(L_{Q}\right)(d \mu)} \leq C\|w\|_{\phi_{1}\left(L_{Q}\right)(d \mu)}
$$

and so property (b) of $G_{1}$ and Corollary 3.16 together imply that $w \in A_{\infty}(d \mu)$, as required. If $p_{1}=1$, then we can argue as in Proposition 3.14 that $w \in A_{\infty}$ (in Proposition 3.14, we assumed $\phi_{1}$ is convex, but we only used convexity to prove that $f_{Q} \hat{w} \leq C$, where $\hat{w}=w /\|w\|_{\phi_{1}\left(L_{Q}\right)(d \mu)}$, a fact that follows easily from property (a) of $G_{1}$ ).

Conversely, if $w \in A_{\infty}(d \mu)$, then $w \in R H_{p}(d \mu)$ for some $p>1$, and so by Lemmas 3.9 and 3.17,

$$
\|w\|_{\phi_{2}\left(L_{Q}\right)(d \mu)} \leq C\|w\|_{L_{Q}^{p}} \leq C\|w\|_{\log \left(L_{Q}\right)(d \mu)} \leq C\|w\|_{\phi_{1}\left(L_{Q}\right)(d \mu)},
$$

as required.

Next, we prove (ii). If (3.19) is true then, by property (a) of $G_{1}$,

$$
\|w\|_{L_{Q}^{p_{2}}(d \mu)} \leq C\|w\|_{\phi_{2}\left(L_{Q}\right)(d \mu)} \leq C\|w\|_{\phi_{1}\left(L_{Q}\right)(d \mu)} \leq C\|w\|_{L_{Q}^{p_{1}(d \mu)}}
$$

and the inequality between the first and last norms implies that $w^{p_{2}} \in A_{r}(d \mu)$, where $r=\left(p_{1}-p_{2}\right) / p_{1}$. Conversely, if $w^{p_{2}} \in A_{r}(d \mu)$, then $w^{p_{2}+\varepsilon} \in A_{r}(d \mu)$, and so

$$
\|w\|_{\phi_{2}\left(L_{Q}\right)(d \mu)} \leq C\|w\|_{L_{Q}^{p_{2}+\varepsilon}(d \mu)} \leq C\|w\|_{L_{Q}^{p_{1}\left(1+e / p_{2}\right)}(d \mu)} \leq C\|w\|_{\phi_{1}\left(L_{Q}\right)(d \mu)} .
$$


Finally, (iii) follows from (i) by taking reciprocal functions $\tilde{\phi}_{i}$, as in the proof of Lemma 3.17.

\section{REFERENCES}

1. A. Besicovitch, A general form of the covering principle and relative differentiation of additive functions, Fund. Math. 41 (1945), 103-110.

2. L. Carleson, On convergence and growth of partial sums of Fourier series, Acta Math. 116 (1966), 135-157.

3. M. Christ and R. Fefferman, A note on weighted norm inequalities for the Hardy-Littlewood maximal operator, Proc. Amer. Math. Soc. 87 (1983), 447-448.

4. R. Coifman and C. Fefferman, Weighted norm inequalities for maximal functions and singular integrals, Studia math. 51 (1974), 241-250.

5. B. Dahlberg, On the Poisson integral for Lipschitz and $C^{1}$ domains, Studia Math. 66 (1979), 7-24.

6. - On the absolute continuity of elliptic measures, Amer. J. Math. 108 (1986), 1119-1138.

7. R. Fefferman, A criterion for the absolute continuity of the harmonic measure associated with an elliptic operator, J. Amer. Math. Soc. 2 (1989), 127-135.

8. R. Fefferman, C. Kenig, and J. Pipher, The theory of weights and the Dirichlet problem for elliptic equations (to appear).

9. J. Garcia-Cuerva and J. Rubio de Francia, Weighted norm inequalities and related topics, Mathematics Studies, North-Holland, 1985.

10. F. Gehring, The $L^{p}$-integrability of the partial derivatives of a quasiconformal mapping, Acta Math. 130 (1973), 265-277.

11. M. de Guzman, Differentiation of integrals in $\mathbf{R}^{n}$, Lecture Notes in Math., vol. 481, Springer-Verlag, 1975.

12. R. Hunt, An estimate of the conjugate function, Studia Math. 44 (1972), 371-377.

13. R. Hunt, B. Muckenhoupt, and R. Wheeden, Weighted norm inequalities for the conjugate function and Hilbert transform, Trans. Amer. Math. Soc. 176 (1973), 227-251.

14. B. Jawerth, Weighted inequalities for maximal operators, Amer. J. Math. 108 (1986), 361414.

15. B. Muckenhoupt, Weighted norm inequalities for the Hardy maximal function, Trans. Amer. Math. Soc. 165 (1972), 207-226.

16. E. Stein, Singular integrals and differentiability properties of functions, Princeton Univ. Press, Princeton, N. J., 1970.

17. J. Strömberg and A. Torchinsky, Weighted Hardy spaces, Lecture Notes in Math., vol. 1281, Springer-Verlag, 1989.

18. A. Zygmund, On a theorem of Marcinkiewicz concerning interpolation of operators, J. Math. 35 (1956), 223-248.

Department of Mathematics, University of Michigan, Ann Arbor, Michigan 48109

Current address: Department of Mathematics, St. Patrick's College, Maynooth, County Kildare, Ireland 